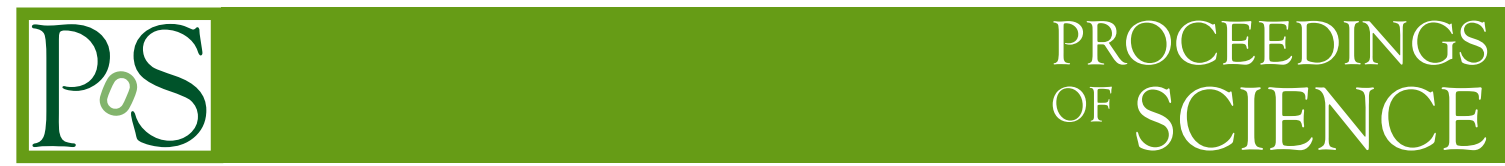

\title{
Preliminary studies on GEM foil degradation in harsh radiation environments
}

Tiago F. da Silva*

Department of Nuclear Physics

University of São Paulo

E-mail: tfsilvaeif.usp.br

\section{Marco Bregant}

Department of Nuclear Physics

University of São Paulo

E-mail: breganteif.usp.br

\section{Geovane G. A. de Souza}

Department of Nuclear Physics

University of São Paulo

E-mail: geovane.souzadusp.br

\section{Cristiane Jahnke}

Department of Nuclear Physics

University of São Paulo

E-mail: cjahnkedif.usp.br

\section{Marcelo G. Munhoz}

Department of Nuclear Physics

University of São Paulo

E-mail: munhoz@if.usp.br 
Several High Energy Physics experiments are adopting Gaseous Electron Multipliers (GEMs) in substitution for the Multi-Wire Proportional Counters (MWPC) either in upgrade plans or in the design stage. Most of the cases, the interest lies in the high count rate capabilities of GEMs together with good spatial resolution. Because of all this interest on GEMs for high count rates measurements, it is important to evaluate the degradation of its constituent materials under such circumstances. This work aims to bring insights from material science to that matter. For this, we studied two possible effects that may play some role in the degradation process of the GEM foils in a harsh radiation environment: One of them is the Kapton degradation due to energy deposition by ionization, which is an effect expected to be triggered by highly energetic particles. We degraded a Kapton foil using 2.2 MeV He+ ions. At this energy, the electronic stopping dominates the interaction processes, thus depositing energy by sequences of ionization. The Kapton composition was assessed by real-time nuclear scattering spectrometry, revealing the Hydrogen losses as the major effect. The second process considered in this study is the erosion of the Copper clad. Opposite to the Kapton degradation, the Copper clad erosion is expected to be induced by low energy ions associated with a sputtering process of the Copper. These low energy ions are expected to be generated during the avalanche of ionization in the amplification process. This effect was explored by computer simulation using the Garfield++ library and estimates of sputtering yields.

7th Annual Conference on Large Hadron Collider Physics - LHCP2019

20-25 May, 2019

Puebla, Mexico

${ }^{*}$ Speaker. 


\section{Introduction}

Several High Energy Physics (HEP) collaborations are adopting Gas Electron Multipliers (GEMs) [1] in substitution for the Multi-Wire Proportional Chambers (MWPC) either in upgrade plans of ongoing experiments or in the design stage of future ones. This is mostly due to limitations of MWPC regarding an upper limit of count rates, which is related to the accumulation of positive ions that imposes the use of a gated mode of operation. Another reason is the spurious currents introduced by the organic deposition in the surfaces of the wires that, as part of a degradation process, reduces the sensitivity and facilitates the occurrence of sparks.

The ALICE experiment, for instance, is passing through a major upgrade that, among other goals, aims the replacement of the MWPC by a stack of four GEMs at the readout planes of its Time Projection Chamber (TPC). The ultimate goal for this replacement is to achieve a higher count rate capability and to avoid the need for the gate mode of operation. The count rate enhancement of the TPC together with the Inner Tracking System (ITS) upgrade shall increase substantially the ALICE efficiency for particle identification [2].

Another example is the upgrade of the CMS experiment where GEM are replacing the MWPC at the Muon chamber end caps. Improvements in the maximum limit of count rate and the Higher Level Trigger are expected by adopting a stack of three GEM-foils [3].

Given all this interest on GEMs for high count rates measurements, it is important to evaluate the degradation of its constituent materials under such circumstances. GEMs indeed present much less aging effects when compared to other concepts, like the MWPC [4]. Two reasons are usually attributed to that: 1- the gas amplification is confined inside the GEM holes and far from the collecting electrodes, and 2- the formation of organic deposits occurs in the vicinity of the holes and far from the collecting electrodes. However, other problems like loss of electrical insulation of the Kapton foil and charging up are effects that show some dependence with the time of usage of the detector.

The effects on the mechanical properties of the GEM foil due to radiation was previously studied. Tensile tests on Kapton and GEM foils irradiated with gamma-rays and neutrons revealed modifications on their mechanical properties, which are more pronounced for the Kapton case, and are slightly stronger if moisture is absorbed within the Kapton [5]. This probably indicates some recombination process following a radiation-induced molecular breakup that includes the water molecules to locally change the Kapton composition. Even though the mechanical properties of the GEM foil are dominated by the properties of the copper clad, composition variation on the Kapton part may have some important influence on the electrical properties of the GEM foil, and this effect lacks for systematic studies.

Additionally, charging up effects and the formation of organic deposition in the vicinity of the hole were already reported $[6,7]$, but how these deposits are formed and how it may affect the charging up is still not clear.

This paper reports the current status of an initiative that aims to bring new insights from material science to that matter. We studied two possible effects that may play some role in the degradation process of the GEM foils in a harsh radiation environment: one of them is the Kapton degradation due to energy deposition by ionization, which is an effect expected to be triggered only by highly energetic particles crossing the foil; the second process considered is the degradation of 
the copper clad. Opposite to the Kapton degradation, the copper clad degradation may be induced by low energy ions associated to a sputtering process of the copper or in a material deposition on its surface, i.e. organic deposition, either from carbon dioxide reduction or sub-products of Kapton degradation.

\section{Methodology}

Aiming at the determination of the most important parameters that influence the detector lifetime, we established a model for the degradation of a GEM foil under a harsh radiation environment. The model consists of two separate processes: one for the degradation of the Kapton substrate, and another for the copper clad.

In our model, the Kapton degradation is expected to occur involving high energy particles crossing the foil, which induces ionization followed by the polymer molecular breakup. During this process, either outgassing of volatile products (e.g. $\mathrm{H}_{2}, \mathrm{CO}, \mathrm{CO}_{2}, \mathrm{O}_{2}, \mathrm{~N}_{2}$, etc.) or crosslinking may occur, changing properties of the Kapton.

Moreover, the degradation of the copper clad is expected to occur involving low energy ions produced in the avalanche process, which may trigger a sputtering process of the copper or start a process for material deposition on its surface by adsorption. One point that is a target for this project is to determine if the deposition in the vicinity of the hole is only related to the products of the $\mathrm{CO}_{2}$ breakup during the avalanche, or if the outgassed volatile products of the Kapton degradation mentioned above play some role in this process.

Figure 1 shows a schematic of the model, indicating the two processes considered.

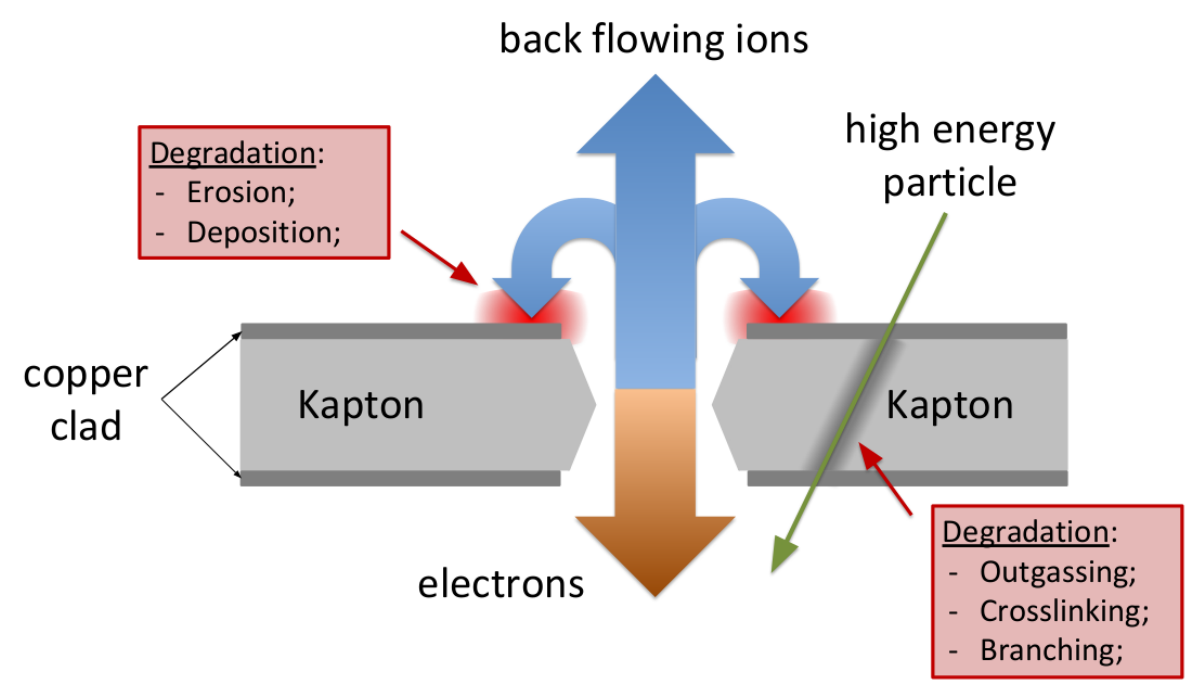

Figure 1: Model of GEM foil degradation. Highly energetic particles can degrade the Kapton substrate by ionization followed by molecular breakup. A fraction of low energy ions produced in the cascade hit the upper copper clad, possibly leading to an erosion process or deposition of organic materials.

To test this model, some preliminary studies were performed. Initially, the Kapton degradation was explored using $2.2 \mathrm{MeV} \mathrm{He}+$ ions. At this energy, the degradation occurs by ionization similarly to the case of high energy particles that cross the foils in HEP experiments. The difference 
is that with this choice of ion and energy, the process is accelerated since the electronic stopping power is higher.

The assessment of the influence of low energy ions in the degradation of the foils was done by computer simulations. We used the Garfield++ framework to map the collisions of ions generated during the avalanche in the vicinity of the holes in the GEM foil. The energy of the ion right before the collision was recorded into the simulation and compared to the expected energies that induces the sputtering of the copper clad.

\section{Results}

\subsection{Results on Kapton degradation}

We degraded a Kapton foil using 2.2 MeV He+ ions. At this energy, the electronic stopping dominates the interaction processes, thus depositing energy by sequences of ionization. The Kapton composition was assessed by real-time nuclear scattering spectrometry, revealing the hydrogen losses as the major effect (see Fig. 2). It is expected that the mechanism of hydrogen loss involves the outgassing of $\mathrm{H}_{2}$ molecules [8]. Oxygen loss is expected in the form of out-gassing of either $\mathrm{CO}$ or $\mathrm{CO}_{2}$ molecules.

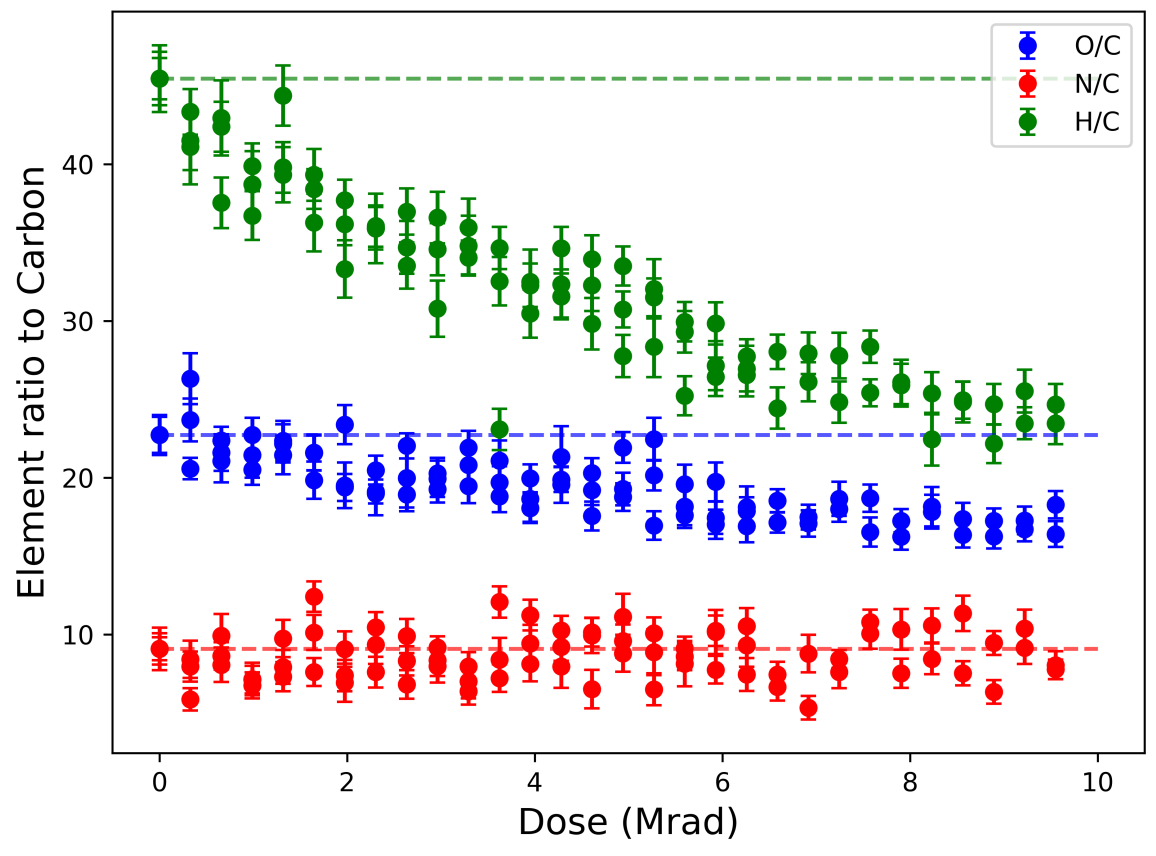

Figure 2: Helium induced Kapton degradation. After the molecular breakup, the products may rearrange into stable gaseous molecules that may outgas. Ratios of oxygen, nitrogen and hydrogen to carbon amounts as a function of absorbed dose. The dashed lines represent the ratios for the pristine Kapton $\left(\mathrm{C}_{22} \mathrm{O}_{5} \mathrm{~N}_{2} \mathrm{H}_{12}\right)$.

\subsection{Results on copper clad erosion}

Part of the low energy ions generated during the avalanche process is expected to hit the upper surface of the GEM foil (see schematic in Fig. 1). This effect was simulated using the Garfield++ 
framework [9]. Fig. 3 (a) shows a map of hits distribution of Argon ions; Fig. 3 (b) shows the mean kinetic energy of the Argon ions right before hitting the GEM foil surface.

(a)

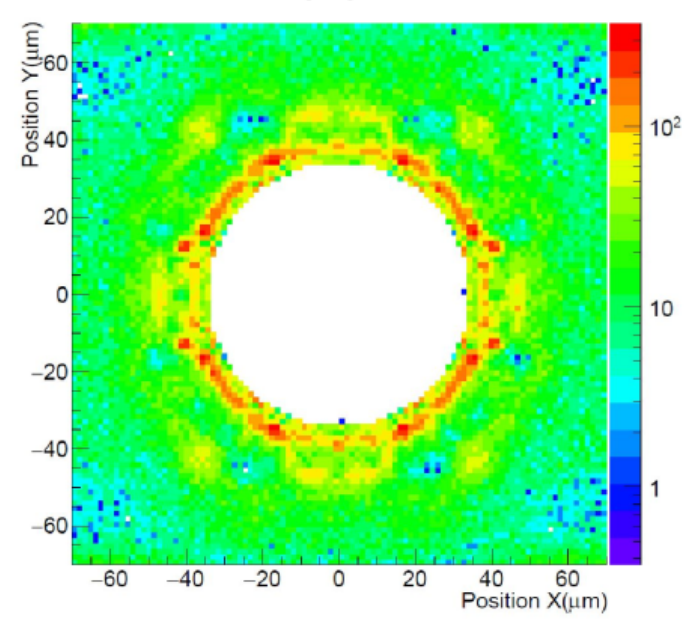

(b)

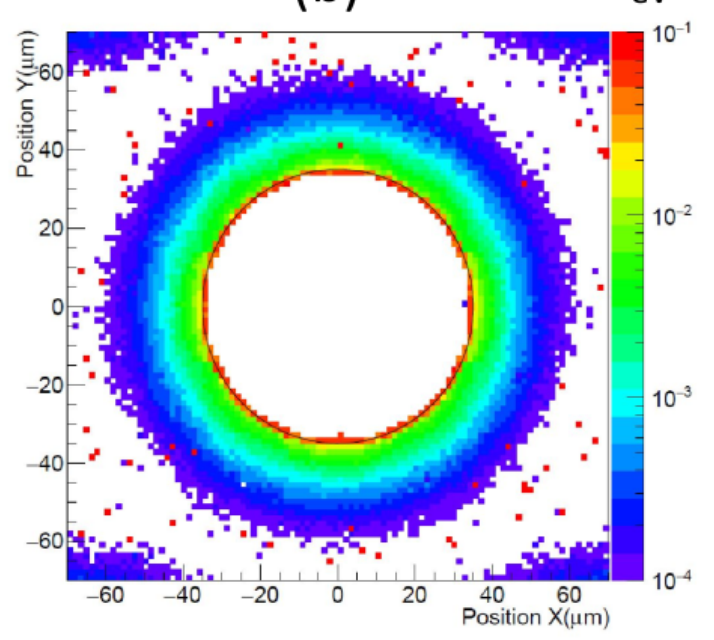

Figure 3: Argon ions hits at the upper surface of a GEM foil as simulated using Garfield++ framework. (a) Map of hits distribution; (b) Mean kinetic energy of Argon ions right before hitting the surface.

\section{Discussion and conclusions}

In this preliminary study, we contemplated the degradation process of GEM foils when submitted to a harsh radiation environment. We considered the Kapton substrate and the copper clad degradation separately: while the Kapton degradation by high energy particles was studied experimentally using a $2.2 \mathrm{MeV} \mathrm{He}{ }^{+}$beam, the copper clad degradation by the low energy ions was explored through simulations.

For the Kapton case, the most pronounced effect observed was a high rate of hydrogen loss. As a molecule with high dissociation energy, it is reasonable to assume the formation of $\mathrm{H}_{2}$ after the polymer molecular breakup during the degradation process. These $\mathrm{H}_{2}$ molecules outgas and dilute into the detector's gas. The concentration of $\mathrm{H}_{2}$ within the detector will depend on the rate of gas renewal.

Fig. 4 shows estimates for the volume of $\mathrm{H}_{2}$ released as a function of the deposited dose, based on the measurements shown in Fig. 2. These estimates were calculated for standard conditions of temperature and pressure and assuming a $1.0-\mathrm{m}^{2}$ area and $50-\mu \mathrm{m}$ thick GEM foil. The painted region denotes a confidence region for the model of hydrogen release derived by Adel [8], and is used in this plot to show the tendency of the data.

The hydrogen release is an important issue to study since the formation of complex organic deposits on the copper surface only occurs in the presence of $\mathrm{H}_{2}$, and it may be connected to the formation of organic deposits in the vicinity of the hole in GEM foils. This is a complex issue and is still a target of our efforts in a better understanding of the GEM detectors degradation. At least, at the present status of our research, it is possible to establish as a threshold for the $\mathrm{H}_{2}$ release the dose of approximately $200 \mathrm{krad}$. 
For the copper clad case, the maximum energy observed in simulations for the Argon ions generated during the ionization cascade that hits the upper surface of the foil was of the order of $10^{-1} \mathrm{eV}$. This energy is far below the threshold energy for sputtering, which is approximately 27 $\mathrm{eV}$ for the case of Argon incident on copper [10], and consequently not enough to trigger an erosion process of the clad.

As a perspective of this work, we intend to study the organic material deposition at the GEM surface.

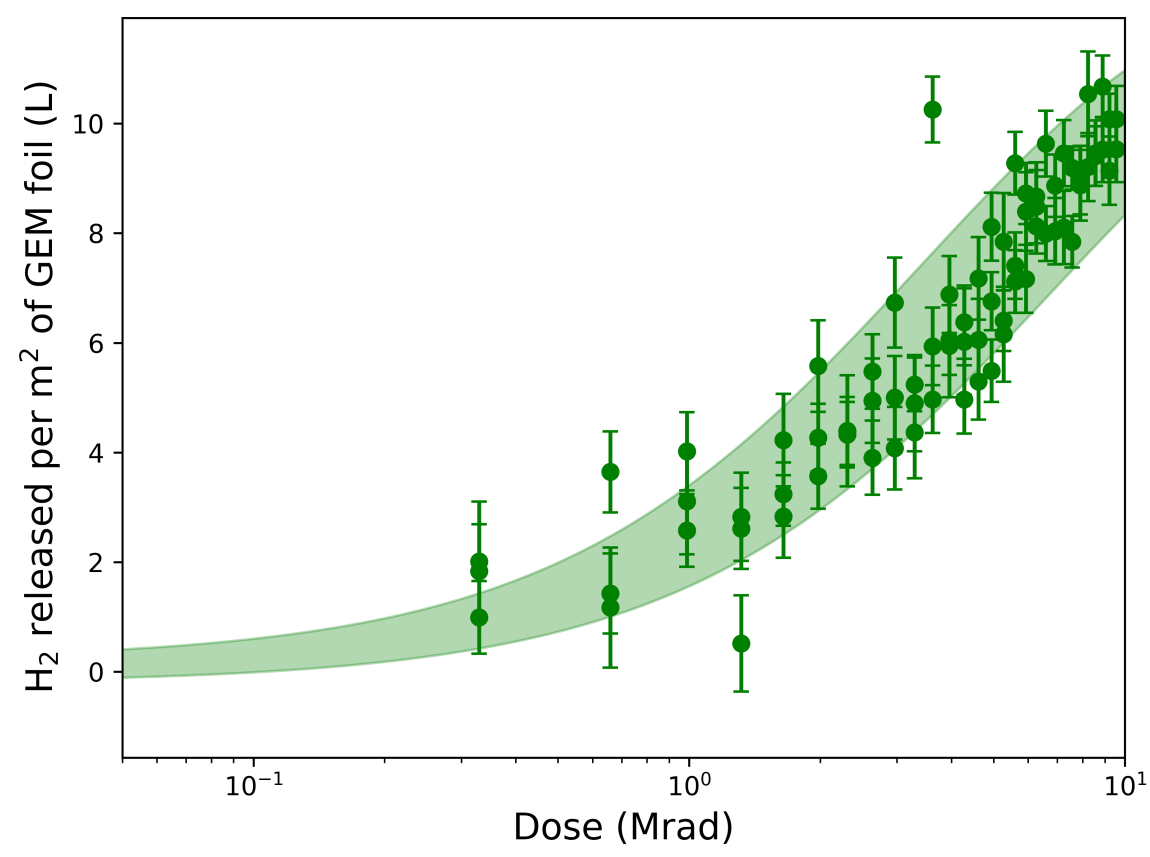

Figure 4: Estimate volume of released $\mathrm{H}_{2}$ at standard conditions for temperature and pressure as function of deposited dose. Values for $1.0-\mathrm{m}^{2}$ area and $50.0-\mu \mathrm{m}$ thick GEM foil.

\section{Acknowledgements}

The authors thank for the real-time scattering spectrometry to the Laboratory of Material Analysis with Ion Beams of the University of São Paulo (LAMFI-USP).

Financial support from FAPESP (project number 12/04583-8 and 2016/05282-2) and CNPq (INCT-FNA 464898/2014-5) Brazilian funding agencies is acknowledged.

\section{References}

[1] F. Sauli, "The gas electron multiplier (GEM): Operating principles and applications", Nuclear Instruments and Methods in Physics Research Section A: Accelerators, Spectrometers, Detectors and Associated Equipment, vol. 805, p. 2-24, jan. 2016.

[2] "Technical Design Report for the Upgrade of the ALICE Time Projection Chamber" (DOI: 10.13140/RG.2.1.1761.7681). 
[3] A. Colaleo, CERN-LHCC-2015-012, 2015.

[4] M. C. Altunbas et al., "Aging measurements with the Gas Electron Multiplier (GEM)", Nuclear Instruments and Methods in Physics Research Section A: Accelerators, Spectrometers, Detectors and Associated Equipment, vol. 515, no. 1-2, pp. 249-254, Dec. 2003.

[5] G. Saviano et al., "A study of mechanical properties of foil materials for the GEM detector proposed for the CMS muon system upgrade at LHC: A Study of Mechanical Properties of Foil Materials for the GEM Detector Proposed for the CMS Muon System Upgrade at LHC", Polym Eng Sci, vol. 58, no. 9, pp. 1539-1547, Sep. 2018.

[6] B. Azmoun et al., "A Study of Gain Stability and Charging Effects in GEM Foils", in 2006 IEEE Nuclear Science Symposium Conference Record, 2006, vol. 6, pp. 3847-3851.

[7] P. S. Barbeau, J. Collar, J. Miyamoto, and I. Shipsey, "An aging study of industrially produced micropatterned gas detectors", in 2003 IEEE Nuclear Science Symposium. Conference Record (IEEE Cat. No.03CH37515), Portland, OR, USA, 2004, pp. 3723-3725.

[8] M.E. Adel et. Al, Journal of Applied Physics 66, 3248 (1989).

[9] H. Schindler, Garfield++, http://garfieldpp.web.cern.ch/garfieldpp/ (May, 2019).

[10] W. Eckstein, "Sputtering Data", IPP REPORT 8/82, 1993. 